\title{
Peguero-Lo Presti criteria modified by body surface area for the electrocardiographic diagnosis of left ventricular hypertrophy in Thai patients
}

\author{
Wongsakorn Luangphiphat ${ }^{\oplus}$, Atthaphon Phaisitkriengkrai ${ }^{\circledR}$, Ratikorn Methavigul ${ }^{\odot}$, \\ Komsing Methavigul*®
}

\begin{abstract}
Background: Several electrocardiographic (ECG) criteria are used to diagnose left ventricular hypertrophy (LVH); however, they have low sensitivity.

Objective: To assess the sensitivity of LVH diagnosis using Peguero-Lo Presti criteria modified by body surface area (BSA).

Methods: This study used retrospective data from 9,438 patients who attended the Central Chest Institute of Thailand from January 2017 to December 2017 with available echocardiography, and who were categorized into those with and without LVH to determine diagnostic accuracy. We randomly selected 317 patients after excluding others based on various conditions. The left ventricular mass of the 317 patients was estimated using echocardiography. Peguero-Lo Presti criteria were modified by dividing original criteria by BSA. The accuracy of the modified criteria was compared with that of the original Peguero-Lo Presti, Sokolow-Lyon, and Cornell voltage criteria. A McNemar test was used to determine the agreement of all ECG criteria examined with LV mass index. The area under a receiver operating characteristic curve (AUC) was used to assess the performance of the criteria.

Results: LVH was diagnosed in 164 of the 317 patients using echocardiography. The sensitivity of modified Peguero-Lo Presti criteria was $50.6 \%$ (95\% confidence interval [CI] $42.7 \%$ to $58.5 \%$ ), and specificity was $88.2 \%$ (95\% CI $82.0 \%$ to 92.9\%), with an AUC of 0.67 (95\% CI 0.61-0.73).

Conclusions: Peguero-Lo Presti criteria modified by dividing them by BSA can improve sensitivity with acceptable specificity for the diagnosis of LVH compared with other ECG criteria examined, at least in selected Thai patients. The modified Peguero-Lo Presti criteria have accuracy similar to that for the original criteria.
\end{abstract}

Keywords: hypertrophy; left ventricular; left ventricular dysfunction; point-of-care testing; predictive value of tests; sensitivity and specificity

Patients with left ventricular hypertrophy (LVH) are common in clinical practice, with a prevalence of $28 \%-62 \%$ in the elderly Thai population [1]. The prevalence of LVH is associated with hypertension, advanced age, obesity, ancestry, and sex $[1,2]$. The regression of electrocardiographic (ECG)
LVH can reduce cardiovascular risk, whereas serial worsening imposes increased cardiovascular risk [3].

ECG criteria have been compared with echocardiographic criteria to diagnose LVH $[4,5]$. When LVH is diagnosed using echocardiography, various methods are used to index

*Correspondence to: Komsing Methavigul, Department of Cardiology, Central Chest Institute of Thailand, Nonthaburi 11000, Thailand, email: komsing@ccit.mail.go.th

Department of Cardiology, Central Chest Institute of Thailand, Nonthaburi 11000, Thailand.

¿ Open Access. ๑ 2021 Luangphiphat et al., published by Sciendo. (Gc)Br-Nc-ND This work is licensed under the Creative Commons Attribution NonCommercial-NoDerivatives 4.0 License. 
LV mass, including the cube, truncated ellipsoid, area-length, and 3D-based formulas, which allow comparisons between individuals with different body sizes [6]. However, echocardiography is not recommended for routine screening for LVH because evidence for an improved cost-benefit ratio is lacking. An ECG is a simpler method with a lower cost for screening for $\mathrm{LVH}$ in the general population, especially in patients at risk for LVH. ECG criteria for diagnosis of LVH usually have high specificity, but low sensitivity [6], such as Sokolow-Lyon voltage criteria, which only have $22 \%$ sensitivity, but specificity as high as $100 \%$. By contrast, Cornell voltage criteria have higher sensitivity (42\%) and specificity (96\%) [7]. Voltageduration products calculated using the Cornell product (multiplying Cornell voltage by QRS duration) and 12-lead QRS sum (multiplying the 12-lead sum of voltage by QRS duration) improved the sensitivity to $37 \%$ (vs. Cornell voltage $28 \%$ ) and $50 \%$ (vs. $12-$ lead voltage $43 \%$ ), respectively $[8,9]$. However, several factors influence the sensitivity for LVH diagnosis. The sensitivity is lower in female patients, those of younger age, those who smoke, and obese individuals [10, 11].

Previously accepted LVH ECG criteria have been used to screen patients at risk of $\mathrm{LVH}$, such as those with hypertension or aortic stenosis. Nevertheless, the sensitivity of the aforementioned criteria for LVH screening remains low. The low sensitivity of these criteria means that even if no LVH is determined by ECG, LVH cannot be excluded. Because variation in body size may be a problem for the sensitivity of LVH ECG criteria, the inclusion of a factor for body surface area (BSA) is hypothesized to improve the ECG voltage criteria [6].

An ECG study by Peguero et al. [12] showed that the sum of the depth of the deepest $\mathrm{S}$ wave in any single lead and the depth of the $\mathrm{S}$ wave in lead V4 $\left(\mathrm{S}_{\mathrm{D}}+\mathrm{SV}_{4}\right)$ could improve sensitivity to $62 \%$ for LVH diagnosis in a small cohort of American patients with a diagnosis of hypertensive crisis and an equal number with normal blood pressure at hospital admission, with a specificity of $90 \%$. The LVH ECG criteria proposed by Peguero et al. [12] (Peguero-Lo Presti criteria) have higher sensitivity for LVH diagnosis than previously used ECG criteria. However, the Peguero-Lo Presti criteria and other previously accepted LVH ECG criteria do not include body weight, body mass index (BMI), or BSA. Moreover, there is limited sensitivity in Asian patients compared with patients of European ancestry, although modified sex-specific cut-offs may help to improve sensitivity while preserving specificity [13].

Patients with higher body weight, BMI, or BSA may have a smaller QRS. This is a common problem in the voltage ECG criteria for the diagnosis of LVH. Therefore, we modified the original Peguero-Lo Presti criteria by dividing by BSA and hypothesized that this would improve their sensitivity for LVH diagnosis in patients with Thai ancestry.

\section{Methods}

The present study was approved by the Human Research Ethics Committee of the Central Chest Institute of Thailand (approval No. 117/2561), was conducted in accordance with the principles outlined in the contemporary revision of the Declaration of Helsinki of 1964 (World Medical Association) incorporating the most recent (2013) and earlier amendments, and was reported according to the STARD 2015 list of essential items for reporting diagnostic accuracy studies [14]. This study of diagnostic accuracy used retrospective data from 9,438 patients who attended the Central Chest Institute of Thailand with available echocardiography, and electrocardiography (ECG) obtained from January 2017 to December 2017 and data were stored in the Institute database. Data from 4,026 patients $(42.7 \%)$ were excluded because ECG showed right ventricular hypertrophy (RVH), complete left bundle branch block, complete right bundle branch block, left anterior fascicular block, left posterior fascicular block, ventricular paced rhythm, second degree atrioventricular block (AVB), complete AV block, patients with pulmonary hypertension, hypertrophic cardiomyopathy, moderate to large pericardial effusion, pneumothorax, pleural effusion, or myocardial infarction within 1 month before echocardiography or ECG recording, LV systolic dysfunction, and pregnancy. After web-based random sampling (https://www.sealedenvelope.com/simple-randomiser/v1/lists), data from 317 patients were included (Figure 1).

The LV mass of all 317 patients whose data were included in the present study was estimated using echocardiography and assessed using the cube formula specified by 2015 recommendations for cardiac chamber quantification by echocardiography in adults from the American Society of Echocardiography (ASE) and the European Association of Cardiovascular Imaging (EACVI) [6]. Data from patients with a consensus of echocardiographic LVH by 2 cardiologists were included independently. The ECGs within 6 months of echocardiography were reviewed for all patients who were categorized as patients with and without LVH. The study by Peguero et al. [12] showed an average BSA $1.87 \mathrm{~m}^{2}$ and an $\mathrm{S}_{\mathrm{D}}+\mathrm{SV}_{4} \geq 2.3 \mathrm{mV}$ for female patients and $\geq 2.8 \mathrm{mV}$ for male patients diagnosed as LVH. Peguero-Lo Presti criteria were modified by dividing the criteria by BSA; so, $\mathrm{S}_{\mathrm{D}}+\mathrm{SV}_{4}$ was indexed according to $\mathrm{BSA} \geq 1.5 \mathrm{mV} / \mathrm{m}^{2}$ for male patients and $\geq 1.2 \mathrm{mV} / \mathrm{m}^{2}$ for female patients and considered a diagnosis for LVH. Two cardiologists (WL and AP) assessed ECG criteria independently, including modified Peguero-Lo Presti criteria, Peguero-Lo Presti criteria, Cornell voltage criteria, and Sokolow-Lyon criteria for all patients. There was a high reproducibility as reflected by an intraclass correlation $>0.90$ for all ECG criteria. 


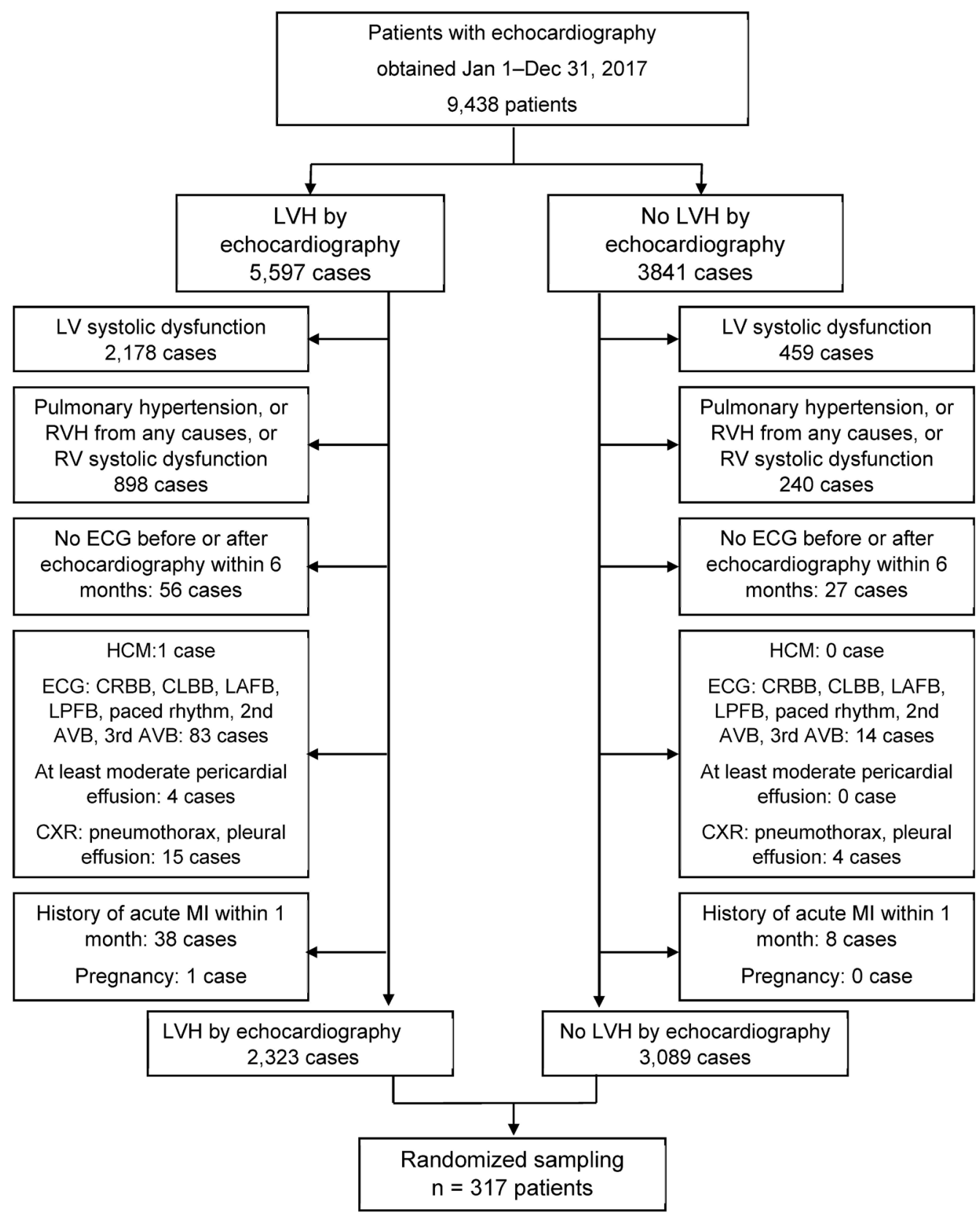

Figure 1. Flow chart for sampling of patients whose data were included in the study. Randomized sampling was web-based (https://www. sealedenvelope.com/simple-randomiser/v1/lists). AVB, atrioventricular block; CXR, chest X-ray imaging; CRBB, complete right bundle branch block; CLBB, complete left bundle branch block; ECG, electrocardiography; HCM, hypertrophic cardiomyopathy; LV, left ventricular; LVH, left ventricular hypertropy; LAFB, left anterior fascicular block; LPFB, left posterior fascicular block; MI, myocardial infarction; RV, right ventricular; $\mathrm{RVH}$, right ventricular hypertrophy.

\section{Echocardiographic analysis}

Transthoracic echocardiography had been performed using EPIQ 5 or 7, and IE33 cardiovascular ultrasound systems (Philips). Patients had lain in a partial left lateral decubitus position under stable hemodynamic conditions. Interventricular septum thickness, LV internal diameter, and inferolateral wall thickness had been acquired at enddiastole using a parasternal approach, perpendicular to the LV long axis. LV mass was calculated using a linear method (cube formula), either in M-mode or 2D tracing, and indexed according to BSA. LVH was diagnosed if LV mass index was $>115 \mathrm{~g} / \mathrm{m}^{2}$ in male patients and $>95 \mathrm{~g} / \mathrm{m}^{2}$ in female patients [5]. 


\section{ECG analysis}

Standard 12-lead ECGs had been performed in all patients with normal calibration $(10 \mathrm{~mm} / \mathrm{mV})$ and speed $(25 \mathrm{~mm} / \mathrm{s})$. The ECG criteria used to diagnose LVH were:

1. Peguero-Lo Presti criteria using a summation of the amplitude of the deepest $\mathrm{S}$ wave in any single lead $\left(\mathrm{S}_{\mathrm{D}}\right)$ and amplitude of $\mathrm{S}$ wave in $\mathrm{V}_{4}\left(\mathrm{SV}_{4}\right)$. LVH is diagnosed when $\mathrm{S}_{\mathrm{D}}+\mathrm{SV}_{4} \geq 2.8 \mathrm{mV}$ in male patients or $\geq 2.3 \mathrm{mV}$ in female patients [12].

2. Modified Peguero-Lo Presti criteria using $\mathrm{S}_{\mathrm{D}}+\mathrm{SV}_{4}$ from Peguero-Lo Presti criteria were indexed according to BSA. LVH was diagnosed when $\mathrm{S}_{\mathrm{D}}+\mathrm{SV}_{4}$ were indexed according to $B S A \geq 1.5 \mathrm{mV} / \mathrm{m}^{2}$ in male patients or $\geq 1.2 \mathrm{mV} /$ $\mathrm{m}^{2}$ in female patients.

3. Sokolow-Lyon voltage criteria using a summation of the amplitude of the $\mathrm{S}$ wave in $\mathrm{V}_{1}$ and the amplitude of $\mathrm{R}$ wave in $\mathrm{V}_{5}$ or $\mathrm{V}_{6}\left(\mathrm{SV}_{1}+\mathrm{RV}_{5}\right.$ or $\left.\mathrm{RV}_{6}\right)$ or the amplitude of the $\mathrm{R}$ wave in aVL (RaVL). $\mathrm{LVH}$ was diagnosed when $\mathrm{SV}_{1}+\mathrm{RV}_{5}$ or $\mathrm{RV}_{6} \geq 3.5 \mathrm{mV}$ or $\mathrm{RaVL} \geq 1.1 \mathrm{mV}[15,16]$.

4. Cornell voltage criteria using a summation of the amplitude of the $\mathrm{S}$ wave in $\mathrm{V}_{3}$ and the amplitude of the $\mathrm{R}$ wave in $\mathrm{aVL}\left(\mathrm{SV}_{3}+\mathrm{RaVL}\right)$. $\mathrm{LVH}$ was diagnosed with $\mathrm{SV}_{3}+\mathrm{RaVL}$ $>2.8 \mathrm{mV}$ in male patients or $>2.0 \mathrm{mV}$ in female patients $[7,15,17]$.

\section{Statistical analysis}

We specified 0.05 for a type I error and estimated 0.57 as a proportion for sensitivity (modified from Peguero et al. [12]). An acceptable error was considered as $18 \%$ of sensitivity. The estimated prevalence of $\mathrm{LVH}$ was $28 \%$ (from Chantra and Bhuthong [1]). We estimated a required sample size of 317 patients.

Categorical data are presented as frequency and percentage. Continuous variables are presented as mean \pm standard deviation (SD). Sensitivity, specificity, positive predictive value (PPV), and negative predictive value (NPV) were calculated for all ECG criteria presented. A McNemar test was used to confirm the agreement of all ECG criteria presented against the LV mass index. The area under the receiver-operating characteristic curve (AUC) was used to assess the performance of the criteria. Cronbach $\alpha$ was used to determine interobserver variability between the 2 independent cardiologists. $P<0.05$ was considered significant.

\section{Results}

The average age of patients whose data were included in the present study was $57.2 \pm 16.5$ years. Their average BSA was
$1.7 \pm 0.2 \mathrm{~m}^{2}$. Nearly $50 \%$ had hypertension. About $20 \%$ of patients had atrial fibrillation and about $25 \%$ of patients had valvular heart disease. Of those, 164 patients had LVH and 153 patients did not. Compared with patients without LVH, more patients with LVH had advanced age, hypertension, diabetes mellitus, dyslipidemia, heart failure, and higher systolic blood pressure. The average left ventricular ejection fraction in patients with LVH was $66.1 \pm 7.5 \%$ and $67.1 \pm 7.1 \%$ in those without. The average LV mass index in patients with $\mathrm{LVH}$ was $141.6 \pm 35.9 \mathrm{~g} / \mathrm{m}^{2}$ and it was $82.3 \pm 17.3 \mathrm{~g} / \mathrm{m}^{2}$ in those without LVH. Demographic data indicating baseline characteristics of patients with and without LVH are shown in Table 1.

The sensitivity of modified Peguero-Lo Presti criteria was $50.6 \%$ (95\% confidence interval [CI] $42.7 \%$ to $58.5 \%$ ), specificity was $88.2 \%$ (95\% CI $82.0 \%$ to $92.9 \%$ ), PPV was $82.2 \%$ (95\% CI $73.3 \%$ to $89.1 \%$ ), and NPV was $62.5 \%$ (95\% CI $55.7 \%$ to $69.0 \%$ ). The modified Peguero-Lo Presti criteria had the best sensitivity and acceptable specificity compared with the original Peguero-Lo Presti criteria, Sokolow-Lyon voltage criteria, and Cornell voltage criteria (Table 2 and Figure 2). Compared with the original Peguero-Lo Presti criteria, the modified Peguero-Lo Presti criteria had comparable accuracy with an AUC of 0.67 (95\% CI $0.61-0.73 ; P<0.01$; Table 2 and Figure 3).

\section{Discussion}

The present study shows that Peguero-Lo Presti criteria modified by BSA can improve their sensitivity for the diagnosis of LVH with specificity and accuracy compared with original Peguero-Lo Presti criteria. Moreover, the modified criteria had better sensitivity and similar specificity than previously accepted ECG criteria for LVH, such as Sokolow-Lyon voltage criteria or Cornell voltage criteria. Height and body weight are required to calculate BSA to interpret $\mathrm{LVH}$ voltage ECG criteria.

The sensitivity of all existing ECG criteria presented in the present study was lower than that found by previous studies $[7,12,15]$. There are several possible explanations underlying the observation that the sensitivity was lower than expected. First, previous studies recruited healthy patients for the control group, while the present study included data from diseased patients as controls were decreasing in sensitivity. Second, previous studies used a higher LV mass index for diagnosis of LVH, while this study used a lower LV mass index following the 2015 ASE-EACVI recommendations for cardiac chamber quantification by echocardiography in adults [6] and improved accuracy of echocardiographic LVH as reflected by an intraclass correlation $>0.90$ in all ECG criteria. Finally, people of Asian ancestry, including Thais, may have a body size and 
Table 1. Baseline characteristics of the patients with available echocardiography and with and without LVH

\begin{tabular}{|c|c|c|c|c|}
\hline Demographic & $\begin{array}{c}\text { Total } \mathbf{n}=\mathbf{3 1 7} \\
\mathrm{n}(\%) \text { or mean } \pm \text { SD }\end{array}$ & $\begin{array}{c}\text { LVH } n=164 \\
n(\%) \text { or mean } \pm \text { SD }\end{array}$ & $\begin{array}{c}\text { No LVH } n=153 \\
n(\%) \text { or mean } \pm \text { SD }\end{array}$ & $P$ \\
\hline Age (years) & $57.2 \pm 16.5$ & $63.5 \pm 13.2$ & $50.4 \pm 17.1$ & $<0.01$ \\
\hline Male sex & $134(42.3 \%)$ & $59(36 \%)$ & 75 (49\%) & 0.02 \\
\hline Body weight (kg) & $64.6 \pm 15.2$ & $64.6 \pm 15.9$ & $64.5 \pm 14.5$ & 0.98 \\
\hline Body surface area $\left(\mathrm{m}^{2}\right)$ & $1.7 \pm 0.2$ & $1.7 \pm 0.2$ & $1.7 \pm 0.2$ & 0.37 \\
\hline \multicolumn{5}{|l|}{ Medical history } \\
\hline Hypertension & $151(48)$ & $97(59)$ & $54(35)$ & $<0.01$ \\
\hline Diabetes mellitus & $53(17)$ & $36(22)$ & $17(11)$ & 0.01 \\
\hline Dyslipidemia & $158(50)$ & $101(62)$ & $57(37)$ & $<0.01$ \\
\hline COPD & $16(5)$ & $11(7)$ & $5(3)$ & 0.16 \\
\hline Heart failure & $58(18)$ & $42(26)$ & $16(11)$ & $<0.01$ \\
\hline Atrial fibrillation & $57(18)$ & $29(18)$ & $28(18)$ & 0.89 \\
\hline Peripheral arterial disease & $3(1)$ & $1(1)$ & $2(1)$ & 0.52 \\
\hline History of myocardial infarction & $20(6)$ & $12(7)$ & $8(5)$ & 0.45 \\
\hline Valvular heart disease & $76(24)$ & $46(28)$ & $30(20)$ & 0.08 \\
\hline • Aortic stenosis & $8(3)$ & $7(4)$ & $1(1)$ & 0.04 \\
\hline - Aortic regurgitation & $13(4)$ & $12(7)$ & $1(1)$ & $<0.01$ \\
\hline - Mitral regurgitation & $15(5)$ & $13(8)$ & $2(1)$ & 0.01 \\
\hline History of stroke or TIA & $12(4)$ & $8(5)$ & $4(3)$ & 0.29 \\
\hline Chronic kidney disease & $10(3)$ & $7(4)$ & $3(2)$ & 0.24 \\
\hline Prior PCl & $22(7)$ & $15(9)$ & $7(5)$ & 0.11 \\
\hline Prior CABG & $8(3)$ & $5(3)$ & $3(2)$ & 0.54 \\
\hline \multicolumn{5}{|l|}{ Echocardiographic parameters } \\
\hline LVEF (\%) & $66.5 \pm 7.3$ & $66.1 \pm 7.5$ & $67.1 \pm 7.1$ & 0.18 \\
\hline LV mass index $\left(\mathrm{g} / \mathrm{m}^{2}\right)$ & $113.0 \pm 41.1$ & $141.6 \pm 35.9$ & $82.3 \pm 17.3$ & $<0.01$ \\
\hline Relative wall thickness & $0.6 \pm 2.3$ & $0.8 \pm 3.2$ & $0.5 \pm 0.1$ & 0.24 \\
\hline \multicolumn{5}{|l|}{ Medications } \\
\hline - Beta-blockers & $95(30)$ & $56(34)$ & $39(26)$ & 0.09 \\
\hline - ACEIs/ARBs & $48(15)$ & $32(20)$ & $16(11)$ & 0.03 \\
\hline - Calcium channel blockers & $71(22)$ & $48(29)$ & $23(15)$ & $<0.01$ \\
\hline • Diuretics & $44(14)$ & $33(20)$ & $11(7)$ & $<0.01$ \\
\hline SBP $(\mathrm{mmHg})$ & $130.1 \pm 20.1$ & $135.2 \pm 20.8$ & $124.6 \pm 17.7$ & $<0.01$ \\
\hline $\mathrm{DBP}(\mathrm{mmHg})$ & $77.1 \pm 13.3$ & $78.1 \pm 13.46$ & $76.1 \pm 13.0$ & 0.20 \\
\hline Creatinine (mg/dL) & $1.0 \pm 0.7$ & $1.1 \pm 1.0$ & $0.9 \pm 0.3$ & 0.23 \\
\hline $\mathrm{eGFR}\left(\mathrm{mL} / \mathrm{min} / \mathrm{m}^{2}\right)$ & $83.4 \pm 24.4$ & $74.5 \pm 24.2$ & $91.4 \pm 21.9$ & $<0.01$ \\
\hline
\end{tabular}

ACEls, angiotensin converting enzyme inhibitors; ARBs, angiotensin receptor blockers; CABG, coronary artery bypass grafting; COPD, chronic obstructive pulmonary disease; DBP, diastolic blood pressure; eGFR, estimated glomerular filtration rate; LVEF, left ventricular ejection fraction; LVH, left ventricular hypertrophy; $\mathrm{PCl}$, percutaneous coronary intervention; SBP, systolic blood pressure; SD, standard deviation; TIA, transient ischemic attack.

shape that differs from those of European or other ancestries. Nevertheless, the specificity was similar to previous studies for all criteria presented.

We acknowledge that the present study has some limitations. The study design was retrospective and observational; so, a selection bias may exist. Nevertheless, we attempted to minimize the selection bias using web-based random sampling and analyzing each ECG and echocardiogram using a consensus by 2 cardiologists. A good intraclass correlation confirmed reproducibility. We excluded medical conditions, including
LV systolic dysfunction, RVH, and other conduction abnormalities. Therefore, the modified Peguero-Lo Presti criteria may be less accurate when applied to patients with the excluded conditions.

\section{Conclusions}

Peguero-Lo Presti criteria can be modified by dividing them by BSA to improve the sensitivity of the criteria with acceptable 
Table 2. McNemar test and AUC for the electrocardiographic criteria against the echocardiographic LVH

\begin{tabular}{|c|c|c|c|c|c|c|c|}
\hline Test & Sensitivity (\%) & Specificity (\%) & PPV (\%) & NPV (\%) & AUC & $\begin{array}{c}P \\
\text { AUC }\end{array}$ & $\begin{array}{c}{ }^{*} P \\
\text { McNemar }\end{array}$ \\
\hline Peguero-Lo Presti LVH & $29.9(23.0-37.5)$ & $90.9(85.1-94.9)$ & $77.8(65.5-87.3)$ & $54.7(48.4-61.0)$ & $0.67(0.61-0.73)$ & $<0.01$ & $<0.01$ \\
\hline $\begin{array}{l}\text { Modified Peguero-Lo } \\
\text { Presti LVH }\end{array}$ & $50.6(42.7-58.5)$ & $88.2(82.0-92.9)$ & $82.2(73.3-89.1)$ & $62.5(55.7-69.0)$ & $0.67(0.61-0.73)$ & $<0.01$ & $<0.01$ \\
\hline Sokolow-Lyon LVH & $18.3(12.7-25.1)$ & $88.9(82.8-93.4)$ & $63.8(48.5-77.3)$ & $50.4(44.2-56.5)$ & $0.59(0.53-0.66)$ & 0.06 & $<0.01$ \\
\hline Cornell LVH & $14.0(9.1-20.3)$ & $98.0(94.4-99.6)$ & $88.5(69.8-97.6)$ & $51.5(45.6-57.4)$ & $0.71(0.65-0.76)$ & 0.08 & $<0.01$ \\
\hline
\end{tabular}

AUC, area under the receiver-operating characteristic curve; $\mathrm{Cl}$, confidence interval; LVH, left ventricular hypertrophy; NPV, negative predictive value; PPV, positive predictive value. Values are presented as percentage $(95 \% \mathrm{Cl})$.

${ }^{*} P<0.05$ indicates lack of agreement.

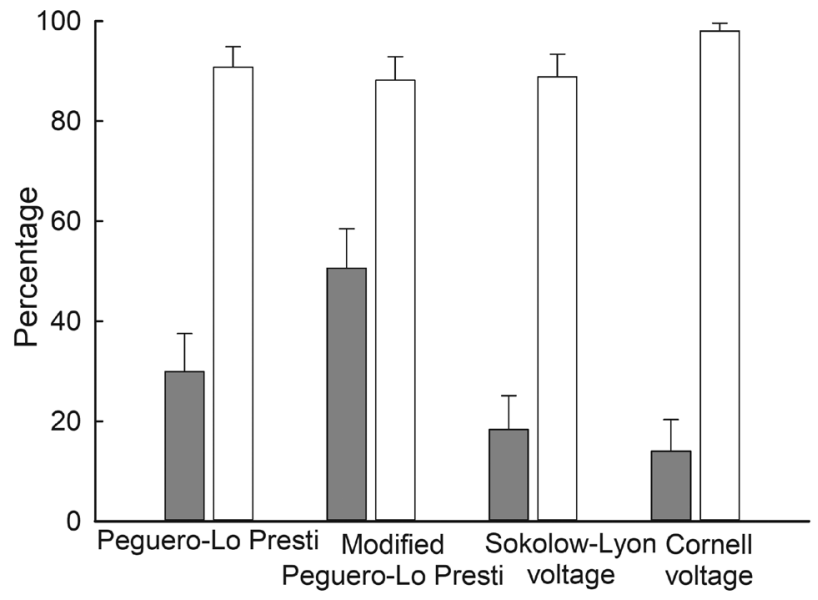

Figure 2. Sensitivity and specificity of the ECG criteria against the echocardiographic LVH. Dark gray bars indicate sensitivity and open bars indicate specificity. Error bars indicate $95 \%$ confidence intervals. ECG, electrocardiographic; LVH, left ventricular hypertrophy.

specificity for the diagnosis of LVH compared with the original Peguero-Lo Presti criteria, Sokolow-Lyon voltage criteria, and Cornell voltage criteria. The modified criteria retain accuracy similar to that of the original Peguero-Lo Presti criteria.

Author contributions: WL and AP contributed substantially to the acquisition of data, WL and KM contributed to the analysis and interpretation of data, and WL, RM, and KM drafted the manuscript. All authors contributed to revise the manuscript critically for important intellectual content. All authors approved the final version submitted for publication and agree to be accountable for all aspects of the work performed in relation to and statements made in the published article.

Acknowledgments: We thank Dollapas Punpanich, Biostatistician at Phramongkutklao Hospital, for sample size calculation and statistical analysis. We did not receive any specific grant for this research from any funding agency in the public, commercial, or not-for-profit sectors.

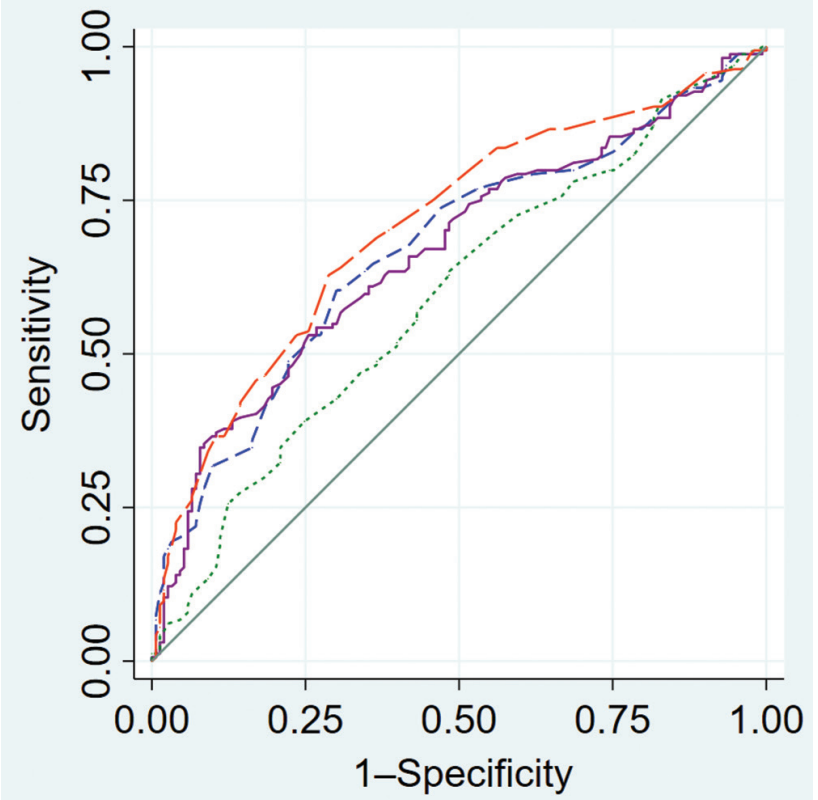

Figure 3. AUC of the ECG criteria against the echocardiographic LVH. Dashed blue line indicates Peguero-Lo Presti criteria (AUC 0.67), solid purple line indicates Peguero-Lo Presti criteria modified by BSA (AUC $0.67)$, short-dashed green line indicates Sokolow-Lyon voltage criteria (AUC 0.59), and long-dashed orange line indicates Cornell voltage criteria (AUC 0.71). The teal line is for reference. AUC, area under a receiver-operating characteristic curve; $B S A$, body surface area; ECG, electrocardiographic; LVH, left ventricular hypertrophy.

Conflicts of interest statement: The authors have each completed an International Committee of Medical Journal Editors Disclosure Form for Potential Conflicts of Interest. None of the authors has disclosed any potential or actual conflict of interest related to the published article.

Data sharing statement: Statistical summaries of the data generated and analyzed for the present study are included in this published article. Further details of the data are available from the corresponding author on reasonable request, after deidentification from any patient. 


\section{References}

[1] Chantra S, Bhuthong B. Echocardiographically detected left ventricular hypertrophy: prevalence and risk factors in Thai elderly men and women. J Med Assoc Thai. 2000; 83:1082-94.

[2] Koncarevic I, Zdravkovic M, Krotin M, Hinic S, Lisulov-Popovic $\mathrm{D}$, Gavrilovic J. Left ventricular hypertrophy in patients with newly diagnosed hypertension: are we late? In: Mancia G, chairman. 25th European Meeting on Hypertension and Cardiovascular Protection; 2015 June 12-15, Milan, Italy: European Society of Hypertension. J Hypertens. 2015; 33(e-Suppl 1):e164. PP.04.08.

[3] Levy D, Salomon M, D’Agostino RB, Belanger AJ, Kannel WB. Prognostic implications of baseline electrocardiographic features and their serial changes in subjects with left ventricular hypertrophy. Circulation. 1994; 90:1786-93.

[4] Tomita S, Ueno H, Takata M, Yasumoto K, Tomoda F, Inoue H. Relationship between electrocardiographic voltage and geometric patterns of left ventricular hypertrophy in patients with essential hypertension. Hypertens Res. 1998; 21:259-66.

[5] Reichek N, Devereux RB. Left ventricular hypertrophy: relationship of anatomic, echocardiographic and electrocardiographic findings. Circulation. 1981; 63:1391-8.

[6] Lang RM, Badano LP, Mor-Avi V, Afilalo J, Armstrong A, Ernande $\mathrm{L}$, et al. Recommendations for cardiac chamber quantification by echocardiography in adults: an update from the American Society of Echocardiography and the European Association of Cardiovascular Imaging. J Am Soc Echocardiogr. 2015; 28:1-39.

[7] Casale PN, Devereux RB, Alonso DR, Campo E, Kligfield P. Improved sex-specific criteria of left ventricular hypertrophy for clinical and computer interpretation of electrocardiograms: validation with autopsy findings. Circulation. 1987; 75:565-72.

[8] Okin PM, Roman MJ, Devereux RB, Kligfield P. Electrocardiographic identification of increased left ventricular mass by simple voltage-duration products. J Am Coll Cardiol. 1995; 25:417-23.

[9] Mahn JJ, Dubey E, Brody A, Welch R, Zalenski R, Flack JM, et al. Test characteristics of electrocardiography for detection of left ventricular hypertrophy in asymptomatic emergency department patients with hypertension. Acad Emerg Med. 2014; 21:996-1002.
[10] Levy D, Labib SB, Anderson KM, Christiansen JC, Kannel WB, Castelli WP. Determinants of sensitivity and specificity of electrocardiographic criteria for left ventricular hypertrophy. Circulation. 1990; 81:815-20.

[11] Hancock EW, Deal BJ, Mirvis DM, Okin P, Kligfield P, Gettes LS, et al.; American Heart Association Electrocardiography and Arrhythmias Committee, Council on Clinical Cardiology; American College of Cardiology Foundation; Heart Rhythm Society. AHA/ACCF/HRS recommendations for the standardization and interpretation of the electrocardiogram: part V: electrocardiogram changes associated with cardiac chamber hypertrophy: a scientific statement from the American Heart Association Electrocardiography and Arrhythmias Committee, Council on Clinical Cardiology; the American College of Cardiology Foundation; and the Heart Rhythm Society. Endorsed by the International Society for Computerized Electrocardiology. J Am Coll Cardiol. 2009; 53:992-1002.

[12] Peguero JG, Lo Presti S, Perez J, Issa O, Brenes JC, Tolentino A. Electrocardiographic criteria for the diagnosis of left ventricular hypertrophy. J Am Coll Cardiol. 2017; 69:1694-703.

[13] Xu CF, Tan ESJ, Feng L, Santhanakrishnan R, Chan MMY, Nyunt SZ, et al. Electrocardiographic criteria for left ventricular hypertrophy in Asians differs from criteria derived from western populations-community-based data from an Asian population. Ann Acad Med Singap. 2015; 44:274-83.

[14] Bossuyt PM, Reitsma JB, Bruns DE, Gatsonis CA, Glasziou PP, Irwig L, et al.; STARD Group. STARD 2015: an updated list of essential items for reporting diagnostic accuracy studies. Clin Chem. 2015; 61:1446-52.

[15] Romhilt DW, Bove KE, Norris RJ, Conyers E, Conradi S, Rowlands DT, Scott RC. A critical appraisal of the electrocardiographic criteria for the diagnosis of left ventricular hypertrophy. Circulation. 1969; 40:185-96.

[16] Sokolow M, Lyon TP. The ventricular complex in right ventricular hypertrophy as obtained by unipolar precordial and limb leads. Am Heart J. 1949; 38:273-94.

[17] Schillaci G, Verdecchia P, Borgioni C, Ciucci A, Guerrieri M, Zampi I, et al. Improved electrocardiographic diagnosis of left ventricular hypertrophy. Am J Cardiol. 1994; 74:714-9. 